\title{
3 Pharmaka aus Anästhesie und Intensivmedizin
}

\author{
Monika Bäumel und Alexander Kratzer
}

Da die Prävalenz der Adipositas in der Bevölkerung weltweit stetig ansteigt, sind Ärzte und Pflegepersonal in Anästhesie und Intensivmedizin in ihrer täglichen Praxis zunehmend mit der Behandlung von fettleibigen Patienten konfrontiert. Bedauerlicherweise werden adipöse Patienten von den meisten klinischen Studien zur Erprobung von Arzneimitteln ausgeschlossen, sodass zu den meisten Pharmaka nur wenige bis keine Daten zur Anwendung an fettleibigen Patienten existieren.

\subsection{Arzneimittel zur Allgemeinanästhesie und Analgosedierung}

\subsubsection{Injektionsnarkosemittel}

\section{Thiopental (z.B. Trapanal $\left.{ }^{\circledR}\right)$}

\section{Charakterisierung}

n Thiopental ist ein Injektionsanästhetikum aus der Cruppe der Barbitursäure-Derivate.

- Thiopental wirkt durch Interaktion mit GABARezeptoren. Die Substanz besitzt keine analgetischen Eigenschaften.

- Thiopental ist zugelassen zur Durchführung von Kurznarkosen ohne Intubation sowie zur Einleitung einer Allgemeinanästhesie mit oder ohne Intubation.

- Thiopental penetriert infolge seiner Lipophilie gut in biologische Membranen und passiert so- mit rasch die Blut-Hirn-Schranke, was den schnellen Wirkeintritt erklärt. Durch seine lipophilen Eigenschaften reichert sich Thiopental im Fettgewebe an .

\section{Übliche Dosierungsangabe}

Die mittlere Dosierung beträgt 3-5 mg Thiopental i.v. pro kg Körpergewicht. Die Dosierung erfolgt streng nach Wirkung.

\section{Besonderheiten bei Adipositas}

Da Thiopental aufgrund seiner Lipophilie ins Fettgewebe penetrieren kann, zeigt sich bei adipösen im Vergleich zu normalgewichtigen Patienten eine Erhöhung des Verteilungsvolumens. Dies führt zu einer deutlichen Verlängerung der Halbwertszeit bei Anwendung von Thiopental bei fettleibigen Menschen. Während die Halbwertszeit bei normalgewichtigen Patienten bei ca. 11,6 h liegt, konnten Jung et al. (1982) in einer klinischen Untersuchung bei adipösen Patienten eine durchschnittliche Halbwertszeit von $27,8 \mathrm{~h}$ ermitteln. Daher ist bei adipösen Patienten mit einem längeren Anhalten der Residualwirkung zu rechnen.

Die Gesamtkörperclearance von Thiopental ist bei adipösen Patienten ebenfalls erhöht. In pharmakokinetischen Untersuchungen konnte gezeigt werden, dass eine Dosierung von Thiopental nach fettfreier Körpermasse adäquate Plasmaspiegel zur Narkoseeinleitung ergibt. 
Allerdings muss davon ausgegangen werden, dass ein adipöser Patient im Vergleich zu einem normalgewichtigen nach einmaliger Bolusgabe von Thiopental schneller aus der Narkose erwacht. Dies ist eine Folge des bei fettleibigen Menschen erhöhten Herzminutenvolumens, da dadurch die Rückverteilung vom ZNS in andere Kompartimente (z.B. Fettgewebe) beschleunigt wird und somit schneller nicht mehr genug Thiopental im ZNS zur Aufrechterhaltung der narkotischen Wirkung zur Verfügung steht.

Hypoventilation und Apnoe als bekannte Nebenwirkungen von Thiopental können bei adipösen $\mathrm{Pa}$ tienten vermehrt auftreteten, da viele adipöse $\mathrm{Pa}$ tienten auch an einem Schlafapnoesyndrom leiden.

Bei adipösen Patienten sollte bei der Berechnung der Thiopental-Dosis die fettfreie Körpermasse verwendet werden.

\section{Propofol (z.B. Disoprivan ${ }^{\circledR}$ )}

\section{Charakterisierung}

- Propofol ist ein Injektionsnanästhetikum dessen chemische Struktur sich vom Phenol ableitet.

- Propofol ist zugelassen als kurzwirksames, intravenöses Anästhetikum zur Narkoseeinleitung sowie Aufrechterhaltung der Allgemeinanästhesie im Rahmen einer totalen intravenösen Anästhesie, ferner zur Sedierung von beatmeten Patienten im Rahmen der Intensivbehandlung.

- Der molekulare Wirkmechanismus von Propofol ist noch nicht vollständig geklärt. Diskutiert werden unspezifische Wirkungen an Lipidmembranen, sowie Interaktion mit GABA-Rezeptoren.

- Propofol ist ein sehr lipophiler Arzneistoff mit sehr schnellem Wirkeintritt und kurzer Wirkdauer. Dieses pharmakokinetische Profil resultiert aus der raschen Penetration durch die BlutHirn-Schranke mit anschließender schneller Rückverteilung in tiefe Kompartimente (z.B. Fettgewebe).

\section{Übliche Dosierungsangabe}

- Dosierung zur Narkoseinleitung: 1,5-2,5 mg i.v. pro kg $\mathrm{KG}$; bei älteren Patienten (über 55 Jahre) sowie Risikopatienten mit kardialer Vorschädigung evtl. Dosisreduktion bis $1 \mathrm{mg}$ pro $\mathrm{kg} \mathrm{KC}$

- Dosierung zur (Analgo-)Sedierung: 1-4 mg i.v. pro kg KC pro Stunde für maximal sieben Tage; bei älteren Patienten (über 55 Jahre) sowie Risikopatienten mit kardialer Vorschädigung Dosisreduktion

\section{Besonderheiten bei Adipositas}

Durch seine physikochemischen Eigenschaften als lipophile Substanz kann Propofol gut ins Fettgewebe eindringen, was sich bei adipösen Patienten in einem entsprechend erhöhten Verteilungsvolumen im Vergleich zu Normalgewichtigen niederschlägt. Ferner ist bei Adipositas auch eine deutlich erhöhte Gesamtkörperclearance von Propofol feststellbar. In klinischen Untersuchungen (Servin et al. 1993) konnte gezeigt werden, dass sowohl die Zunahme des Verteilungsvolumens, als auch der Anstieg der Clearance von Propofol signifikant mit dem Anstieg des Gesamtkörpergewichts korrelieren. Da Clearance und Verteilungsvolumen gleichermaßen mit steigendem Gesamtkörpergewicht zunehmen, lässt sich anhand der Gleichung zur Abschätzung der Halbwertszeit (s. Abb. 5 in Kap. VIII.1.2.3) leicht erklären, dass die Halbwertszeit bei adipösen und normalgewichtigen Patienten identisch ist. So ergaben die pharmakokinetischen Untersuchungen von Servin et al. (1993) für fettleibige und normalgewichtige Testpersonen nahezu identische Halbwertszeiten (29,1 min vs. 24,2 min). Daher wird empfohlen, die Erhaltungsdosis von Propofol zur Aufrechterhaltung einer Narkose nach Gesamtkörpergewicht zu errechnen, wohingegen die Dosis zur Einleitung einer Narkose nach fettfreier Körpermasse gewählt werden sollte, um eventuell zu große initiale Boli zu vermeiden. Unter Anwendung von Propofol kann es besonders initial zu hämodynamischen Nebenwirkungen wie starkem Blutdruckabfall kommen. Adipöse Patienten weisen ein erhöhtes Risiko für derartige Komplikationen auf, was sich auch in einem gesonderten Hinweis diesbezüglich in den Fachinformationen der entsprechenden Propofol-Präparate niederschlägt.

Zur Berechnung der Propofol-Dosis für die Narkoseeinleitung sollte die fettfreie Körpermasse verwendet werden.

Zur Berechnung der Propofol-Dosis zur Aufrechterhaltung einer Narkose sollte das Gesamtkörpergewicht verwendet werden.

\subsubsection{Inhalationsnarkotika}

Zur Verwendung von Inhalationsnarkotika bei adipösen Patienten liegen nur sehr wenige Daten aus klinischen Studien vor. Ältere Substanzen wie Isofluran weisen im Vergleich zu den neueren Substanzen Sevofluran (z.B. Sevorane ${ }^{\circledR}$ ) und Desfluran (z.B. Suprane ${ }^{\circledR}$ ) eine deutlich erhöhte Lipophilie auf. Daher wird dis- 
kutiert, ob der Einsatz stark lipophiler Substanten wie Isofluran durch vermehrte Anreicherung im Fettgewebe die Einleitungs- sowie Erholungsphase der Narkose bei fettleibigen Patienten verlängern kann. Desfluran dagegen, als neuerer, weniger lipophiler Wirkstoff, kann nur in geringem Maße ins Fettgewebe penetrieren, sodass bei dieser Substanz keine relevanten pharmakokinetischen Unterschiede zwischen normalgewichtigen und adipösen Patienten zu erwarten sind. Allerdings beeinflussen diese physikochemischen Unterschiede die Durchführung einer Narkose bei fettleibigen Patienten im klinischen Alltag nicht in relevantem Ausmaß. So konnten Lemmens et al. (2008) im Rahmen einer klinischen Studie zeigen, dass adipöse und normalgewichtige Patienten nach einer Isoflurannarkose vergleichbare Aufwachzeiten aufweisen.

Daher kann nach jetzigem Kenntnisstand keine eindeutige Empfehlung für die Verwendung eines bestimmten Inhalationsnarkotikums bei adipösen Patienten ausgesprochen werden.

\subsubsection{Opioidanalgetika}

\section{Fentanyl (z.B. Fentanyl-Janssen ${ }^{\circledR}$ Injektionslösung)}

\section{Charakterisierung}

- Fentanyl ist ein stark wirksames synthetisches Opioidanalgetikum mit einer im Vergleich zu Morphin 10o-fach stärkeren analgetischen Wirkung.

- Fentanyl Injektionslösung ist zugelassen als analgetische Komponente bei Anästhesien mit Intubation und Beatmung sowie zur Schmerztherapie bei Intensivpatienten. Als transdermales therapeutisches System wird Fentanyl zurTherapie chronischer starker Schmerzen eingesetzt.

- Fentanyl wirkt als reiner $\mu$-Opioid-RezeptorAgonist.

- Fentanyl penetriert durch seine Lipophilie rasch ins ZNS und wird anschließend in periphere Kompartimente (z.B. Fettgewebe) verteilt.

\section{Übliche Dosierungsangabe}

- Bolusgabe: o,5-5 $\mu$ g Fentanyl i.v. pro kg KC

- Kontinuierliche Infusion: o, 5-5 $\mu$ g Fentanyli.v. pro kg KG pro Stunde; je nach erwünschter Wirkung

\section{Besonderheiten bei Adipositas}

Durch seine lipophilen Eigenschaften flutet Fentanyl rasch im ZNS an, woraufhin eine Umverteilung in tiefe Kompartimente (z.B. Fettgewebe) erfolgt, die die Ursache der kurzen Wirkdauer von Fentanyl darstellt. Dieser für die Fentanyl-Kinetik zentrale Prozess der Umverteilung wird wesentlich durch das Herzminutenvolumen des Patienten beeinflusst, da der beschriebene Verteilungsvorgang vom ZNS ins Fettgewebe durch den Blutstrom erfolgt. Collis et al. (2001) konnten nachweisen, dass bei adipösen gegenüber normalgewichtigen Patienten das Herzminutenvolumen erhöht ist. Diese Erhöhung des Herzminutenvolumens korreliert bei adipösen Patienten mit der Zunahme der fettfreien Körpermasse. Da bei fettleibigen Patienten auch ein Anstieg der Clearance für Fentanyl zu verzeichnen ist, der ebenfalls mit der Zunahme der fettfreien Körpermasse korreliert, sollte Fentanyl bei adipösen Patienten nach fettfreier Körpermasse dosiert werden.

Wie auch bei anderen lipophilen Opioiden besteht bei Fentanyl die Gefahr einer Atemdepression infolge der Rückverteilung des Wirkstoffs aus dem Fettgewebe ins Gehirn, insbesondere nach längerer bzw. wiederholter Anwendung. Dieses Risiko ist bei adipösen Patienten deutlich erhöht, da sie über eine größere Körperfettmasse als Speicher für Fentanyl verfügen. Ferner weisen adipöse Patienten zusätzlich häufig respiratorische Begleiterkrankungen wie das Schlafapnoesyndrom auf, was generell das Risiko für eine Atemdepression steigert. Fettleibige Patienten sollten daher post OP einem intensiven respiratorischen Monitoring unterzogen werden.

Zur Berechnung von Initial- und Erhaltungsdosis von Fentanyl sollte die fettfreie Körpermasse verwendet werden.

\section{Sufentanil (z.B. Sufenta $\left.{ }^{\circledR}\right)$}

\section{Charakterisierung}

- Sufentanil ist ein strukturell mit Fentanyl verwandtes synthetisches Opioidanalgetikum. Die analgetische Wirkung von Sufentanil ist ca. 1.00o-fach potenter als die von Morphin und ca. 10-fach potenter als die von Fentanyl.

- Sufentanil ist zugelassen zur Anästhesie bei allen medizinischen Maßnahmen unter endotrachealer Intubation und Beatmung. Die Verwendung kann als analgetische Komponente in Kombinationsnarkosen erfolgen.

- Die Wirkung von Sufentanil wird durch eine hochaffine Bindung an den $\mu$-Opioid-Rezeptor vermittelt.

- Sufentanil ist eine stark lipophile Substanz. 


\section{Übliche Dosierungsangabe}

- Bolusgabe: o,25-0,5 $\mu$ g Sufentanil i.v. pro kg KC

- Kontinuierliche Infusion: $0,2-1 \mu g$ Sufentanil i.v. pro kg KC pro Stunde; je nach erwünschter Wirkung

\section{Besonderheiten bei Adipositas}

Durch seine ausgeprägte Lipophilie weist Sufentanil bei adipösen Patienten im Vergleich zu Normalgewichtigen ein deutlich erhöhtes Verteilungsvolumen auf. Schwartz et al. (1992) konnten im Rahmen einer Studie nachweisen, dass auch die Eliminationshalbwertszeit bei adipösen Patienten deutlich verlängert ist. So war eine im Mittel ca. 50\%ige Verlängerung der Halbwertszeit bei adipösen Patienten zu verzeichnen. Dies ist eine Folge des langsamen Rückstroms des lipophilen Sufentanils aus dem bei adipösen Patienten vermehrt vorhandenen Körperfett. Bei der Clearance hingegen ergaben sich keine Unterschiede zwischen fettleibigen und normalgewichtigen Probanden. Daher wird empfohlen, die Initialdosis nach dem Gesamtkörpergewicht zu errechnen. Die Erhaltungsdosis dagegen sollte nach fettfreier Körpermasse bestimmt werden. Ebenso wie bei der Verwendung von Fentanyl ist auch bei Sufentanil für adipöse Patienten das Risiko respiratorischer Zwischenfälle wie Atemdepression und Hypoxie erhöht.

Zur Berechnung der Sufentanil-Dosis für die Narkoseeinleitung sollte die Gesamtkörpermasse verwendet werden.

Zur Berechnung der Sufentanil-Dosis zur Aufrechterhaltung einer Narkose sollte die fettfreie Körpermasse verwendet werden.

\section{Remifentanil (z.B. Ultiva ${ }^{\circledR}$ )}

\section{Charakterisierung}

- Remifentanil ist ein potentes synthetisches Opioidanalgetikum. Die analgetische Potenz ist ca. 100-200-fach höher als bei Morphin.

- Remifentanil ist zugelassen als Analgetikum zur Einleitung und/oder Aufrechterhaltung der Anästhesie sowie zur Analgesie von beatmeten Patienten in der Intensivmedizin.

- Remifentanil wirkt als reiner $\mu$-Opioid-RezeptorAgonist und zeichnet sich durch eine extrem kurze Wirkdauer aus.

- Im Vergleich zu Fentanyl und Sufentanil weist Remifentanil nur eine sehr geringe Lipophilie auf.
Übliche Dosierungsangabe

- Dosierung zur Narkoseeinleitung: $0,5^{-1} \mu \mathrm{g}$ i.v. pro kg KG pro min.

- Dosierung zur Narkoseaufrechterhaltung: $0,05-1 \mu \mathrm{g}$ i.v. pro kg KG pro min.; Dosierung im Rahmen einer Kombinationsnarkose je nach zusätzlich verwendeten Anästhetika

\section{Besonderheiten bei Adipositas}

Remifentanil weist nur eine geringe Lipophilie auf und penetriert daher nur in geringem Ausmaß ins Fettgewebe. Somit treten auch keine bedeutenden Differenzen bei Verteilungsvolumen und Halbwertszeit zwischen fettleibigen und normalgewichtigen Patienten auf. Die Clearance von Remifenanil erfolgt aufgrund der Esterstruktur des Pharmakons organunabhängig durch unspezifische Plasma-Esterasen. Daher existieren auch keine relevanten Unterschiede in der Clearance der Substanz zwischen adipösen und normalgewichtigen Patienten. Da also Verteilung und Stoffwechsel von Remifentanil außerhalb des Fettgewebes lokalisiert sind, ist es zweckmäßig, die Initialdosis sowie die Erhaltungsdosis unter Verwendung der fettfreien Körpermasse zu berechnen. Diese Herangehensweise konnte durch Egan et al. (1998) bestätigt werden, die in einer klinischen Studie bei Dosierung von Remifentanil nach fettfreier Körpermasse für adipöse und normalgewichtige Probanden identische Plasma-Konzentrationen nachweisen konnten. Eine Dosierung nach Gesamtkörpergewicht hingegen resultiert in supratherapeutischen Plasmaspiegeln, was die Inzidenz von Nebenwirkungen wie Atemdepression, Bradykardie und Hypotension erhöht.

Zur Berechnung der Initial- und Erhaltungsdosis von Remifentanil sollte die fettfreie Körpermasse verwendet werden.

\subsubsection{Benzodiazepine}

\section{Midazolam (z.B. Dormicum ${ }^{\circledR}$ )}

\section{Charakterisierung}

- Midazolam ist ein Sedativum aus der Cruppe der Benzodiazepine.

- Midazolam ist zugelassen zur Analgosedierung vor bzw. während diagnostischer oder therapeutischer Eingriffe, zur Narkose als Prämedikation, Einleitung oder sedierende Komponente bei Kombinationsnarkosen sowie zur Sedierung in der Intensivmedizin. 
- Die sedative Wirkung von Midazolam beruht auf der Bindung des Wirkstoffs an die Benzodiazepin-Bindungsstelle des GABA $_{A}$-Rezeptors. Dadurch kommt es zu einem verstärkten Chlorid-Ionen-Einstrom, der die exzitatorische Wirkung auf das postsynaptische Neuron vermindert.

- Midazolam ist eine äußerst lipophile Substanz und reichert sich daher stark im Fettgewebe an.

\section{Übliche Dosierungsangabe}

- Sedierung: Anfangsdosis 2-2,5 mg i.v.; Titrationsdosis $1 \mathrm{mg}$; Gesamtdosis 3,5-7,5 mg

- Analgosedierung von Intensivpatienten: o, 03-0,2 mg pro $\mathrm{kg} \mathrm{KG}$ pro Stunde

- Die Dosis sollte stets nach Wirkung titriert werden.

\section{Besonderheiten bei Adipositas}

Durch seine ausgeprägte Lipophilie penetriert Midazolam sehr stark ins Fettgewebe. Bei adipösen Patienten führt dies aufgrund der größeren Masse an Körperfett im Vergleich zu Normalgewichtigen zu einer drastischen Vergrößerung des Verteilungsvolumens. Greenblatt et al. (1984) konnten im Rahmen einer klinischen Studie nachweisen, dass bei adipösen Probanden das Verteilungsvolumen für Midazolam etwa um den Faktor drei gegenüber normalgewichtigen Probanden vergrößert ist. Da nach Bolusgabe von Midazolam Verteilungsvorgänge ins tiefe Kompatiment (Fettgewebe) weitaus mehr Einfluss auf Dauer und Intensität des pharmakologischen Effekts ausüben als Elimination und Clearance, steigt infolge des erhöhten Verteilungsvolumens bei fettleibigen Patienten auch die Halbwertszeit entsprechend an. So konnte in oben genannter Studie bei normalgewichtigen Probanden eine Halbwertszeit für Midazolam von durchschnittlich 2,7 h Stunden ermittelt werden, wohingegen die Halbwertszeit bei fettleibigen Probanden bei durchschnittlich 8,4 h lag. Diese Verlängerung der Halbwerstzeit ist ausschließlich Folge des erhöhten Verteilungsvolumens, da bei der Clearance von Midazolam keine relevanten Unterschiede zwischen adipösen und normalgewichtigen Patienten existieren. Daher sollte die Initialdosis von Midazolam unter Verwendung des Gesamtkörpergewichts berechnet werden, um ausreichende Plasmaspiegel zu erzielen. Die Erhaltungsdosis hingegen sollte nach fettfreier Körpermasse bestimmt werden, da die Clearance von Midazolam durch Adipositas nicht nennenswert beeinträchtigt wird. Da Midazolam ebenfalls wie Opioide atemdepressive Eigenschaften aufweist, ist auch hier das Risiko für respiratorische Zwischenfälle bei adipösen Patienten erhöht.
Zur Berechnung der Midazolam-Initialdosis sollte die Gesamtkörpermasse verwendet werden.

Zur Berechnung der Midazolam-Erhaltungsdosis sollte die fettfreie Körpermasse verwendet werden.

Diese Dosierungsempfehlung kann anhand von Ergebnissen aus klinischen Studien auch auf weitere Benzodiazepine (z.B. Diazepam, Lorazepam) übertragen werden.

Durch die hohe Lipophilie kann sich Midazolam, wie auch andere Benzodiazepine, sehr stark im Fettgewebe anreichern (Kumulation), wenn es über längere Zeit (>24 h) kontinuierlich, z.B. über Perfusor, verabreicht wird. Nach Absetzen flutet Midazolam wieder zurück in die Blutbahn und kann so eine nicht-vorhersehbare Wirkverlängerung hervorrufen. Insbesondere fettleibige Patienten weisen durch ihre große Körperfettmasse diesen Depoteffekt verstärkt auf und tragen somit ein höheres Risiko für diese unerwünschte Arzneimittelwirkung. Von der kontinuierlichen Sedierung mittels Perfusor ist bei adipösen Patienten dringend abzuraten!

\section{Exkurs: Respiratorische Komplikationen bei adipösen Patienten}

Für Opioidanalgetika, aber auch für Benzodiazepine sind schwerwiegende respiratorische Nebenwirkungen wie Apnoe und Atemdepression beschrieben. So weisen die Fachinformationen aller hier genannten Opioide Atemdepression als häufig vorkommende Nebenwirkung aus.

Adipöse Patienten sind aufgrund von respiratorischen Begleiteffekten ihrer Adipositas weitaus häufiger von diesen schwerwiegenden Ereignissen betroffen als normalgewichtige Patienten. So waren nach Auswertungen der American Society of Anesthesiologists in 48\% der Fälle von gemeldeten unerwünschten respiratorischen Ereignissen unter Opioid-Applikation adipöse Patienten betroffen.

Bei adipösen Patienten sind infolge der erhöhten Körpermasse sowohl bei körperlicher Aktivität als auch in Ruhe Sauerstoffaufnahme und Kohlendioxidabgabe vergrößert. Da durch Adipositas die Dehnbarkeit der Thoraxorgane vermindert sowie die dem Atemfluss entgegenstehenden Widerstände erhöht sind, ist die Atemarbeit bei Fettleibigkeit deutlich größer. Das Lungenvolumen ist bei Adipositas durch den Druck des Abdomens auf das Zwerchfell verkleinert. Diese Faktoren resultieren schließlich bei adipösen Patienten in einem gegenüber Normal- 
gewichtigen erniedrigten Sauerstoffpartialdruck im Blut. Ferner ist bei Fettleibigkeit auch die Prävalenz der obstruktiven Schlafapnoe sowie des Hypoventilationssyndroms stark erhöht. Daher ist das Risiko einer Atemdepression und der daraus folgenden Hypoxie bei adipösen Patienten unter Anästhesie deutlich erhöht, was ein besonders sorgfältiges respiratorisches Monitoring dieser Patienten verlangt.

\subsubsection{Muskelrelaxantien}

\section{Suxamethonium (z.B. Lysthenon ${ }^{\circledR}$ )}

\section{Charakterisierung}

- Suxamethonium ist das einzige therapeutisch verwendete depolarisierende Muskelrelaxans.

- Suxamethonium ist zugelassen zur Muskelrelaxation im Rahmen der Allgemeinanästhesie. Aufgrund der kurzen Wirkdauer wird es häufig zur endotrachealen Intubation verwendet.

- Der Arzneistoff wirkt als Agonist an den Acetylcholin-Rezeptoren der motorischen Endplatte. Dadurch wird initial durch Depolarisation eine Erregung der Muskelfaser ausgelöst. Durch Aufrechterhaltung der Depolarisation kommt es zur Muskelerschlaffung.

- Durch seine ausgeprägte Hydrophilie penetriert Suxamethonium kaum ins Fettgewebe.

\section{Übliche Dosierungsangabe}

Die intravenöse Einzeldosis von Suxamethonium beträgt 1,0-1,5 mg pro kg KG.

\section{Besonderheiten bei Adipositas}

Suxamethonium tritt wegen seiner ausgeprägten Hydrophilie nicht in nennenswertem Maße ins Fettgewebe über, sondern verteilt sich im Wesentlichen im Extrazellulärraum. Die Verstoffwechselung von Suxamethonium erfolgt organunabhängig durch die im Blutplasma vorkommende Pseudocholinesterase. Adipöse Patienten weisen erhöhte Spiegel dieser Esterase im Blut auf und verfügen zusätzlich über ein im Vergleich zu normalgewichtigen Patienten erhöhtes Extrazellulärvolumen. Daher sollte, um eine hinreichende Wirkung bei Adipositas sicherzustellen, die Dosis hier anhand des Gesamtkörpergewichts errrechnet werden.

Da adipöse Patienten ein erhöhtes Risiko für Hypoxie aufweisen, wird Suxamethonium aufgrund seines schnellen Wirkeintritts und der kurzen Wirkdauer als Mittel der Wahl zur Intubation fettleibiger Patienten angesehen.
Zur Dosisberechnung von Suxamethonium sollte bei adipösen Patienten das Gesamtkörpergewicht verwendet werden.

\section{Pancuronium (z.B. Pancuronium-ratiopharm ${ }^{\circledR}$ )}

\section{Charakterisierung}

- Pancuronium ist ein langwirksames Muskelrelaxans vom kompetitiven/nicht-depolarisierenden Typ.

- Pancuronium ist zugelassen zur Muskelrelaxierung im Rahmen der Allgemeinanästhesie.

- Die Substanz wirkt durch kompetitive Verdrängung von Acetylcholin an den cholinergen Rezeptoren der motorischen Endplatte. Da es selbst keinen Effekt am Rezeptor auslöst, kommt es zu keiner Depolarisation.

- Pancuronium ist stark hydrophil und penetriert daher nicht nennenswert ins Fettgewebe.

\section{Übliche Dosierungsangabe}

- Endotracheale Intubation (mit anschließender OP): 0,1 mg i.v. pro kg KG

- Repetitionsdosis: o,o1-o,02 mg i.v. pro kg KG alle 30 bis 45 Minuten zur vollständigen Aufrechterhaltung der neuromuskulären Blockade

\section{Besonderheiten bei Adipositas}

Durch seine hydrophilen, polaren Eigenschaften kann Pancuronium kaum ins Fettgewebe penetrieren, sondern verteilt sich ausschließlich im Extrazellulärraum. Da bei adipösen Patienten infolge ihres erhöhten Körpergewichts auch der Extrazellulärraum vergrößert ist, konnte gezeigt werden, dass fettleibige Patienten für eine ausreichende muskelrelaxierende Wirkung höhere Dosen Pancuronium benötigen als normalgewichtige Patienten. Tsueda et al. (1978) ermittelten im Rahmen einer klinischen Studie das ideale Körpergewicht als geeigneten $\mathrm{Ge}$ wichtsparameter zur Berechnung der erforderlichen Dosis bei fettleibigen Patienten.

Pancuronium wird teilweise über eine Esterspaltung vermittelt durch unspezifische Plasmaesterasen abgebaut. Diese Abbaureaktion ist in ihrer Kinetik pH-Wert abhängig und kann durch einen erniedrigten Blut-pH im Rahmen einer Azidose verlangsamt werden, was die Wirkdauer von Pancuronium deutlich verlängert. Da fettleibige Patienten aufgrund von Adipositas-assoziierten respiratorischen Einschränkungen im Rahmen einer Narkose ein erhöhtes Risiko für eine $\mathrm{CO}_{2}$-Retention und die damit 
verbundene Azidose aufweisen, kann diese unerwünschte Verlängerung der Wirkdauer bei diesem Patientenkollektiv vermehrt auftreten.

Zur Berechnung der benötigten Pancuronium-Dosis bei Adipositas sollte das ideale Körpergewicht oder die fettfreie Körpermasse verwendet werden.

\section{Atracurium (z.B. Atracurium-hameln $10 \mathrm{mg} / \mathrm{ml}$ )}

\section{Charakterisierung}

- Atracurium ist ein mittellang wirksames nichtdepolarisierendes Muskelrelaxans mit EsterStruktur.

- Atracurium ist zugelassen zur Muskelrelaxation bei endotrachealer Intubation, bei operativen Eingriffen sowie in der Intensivmedizin.

- Atracurium als peripher wirkendes Muskelrelaxans bindet kompetitiv an den Acetylcholinrezeptor der motorischen Endplatte, ohne eine Depolarisation auszulösen. Die Wirkung des Acetylcholins wird dadurch blockiert.

- Atracurium ist eine polare hydrophile Substanz.

\section{Übliche Dosierungsangaben}

- Erleichterung der endotrachealen Intubation: o, 5-0,6 mg i.v. pro kg KC

- Repetitionsdosis: o,1-o,15 mg i.v. pro kg KC

- Dosierung streng nach Wirkung!

\section{Besonderheiten bei Adipositas}

Varin et al. (1990) konnten im Rahmen einer klinischen Studie nachweisen, dass sich Verteilungsvolumen, Clearance und Halbwertszeit bei adipösen und normalgewichtigen Patienten nicht unterscheiden. Dies ist eine Folge des organunabhängigen Metabolismus von Atracurium, der im Wesentlichen durch eine chemische Zerfallsreaktion (HoffmannElimination) und durch Esterspaltung mittels Plasmaesterasen vermittelt wird. Daher wird empfohlen, die Dosis von Atracurium bei fettleibigen Patienten nach idealem Körpergewicht zu wählen, da Dosierung nach Gesamtkörpergewicht bei diesem Patientenkollektiv zu supratherapeutischen Plasmakonzentrationen führt.

Bei Adipositas sollte zur Berechnung der benötigten Atracurium-Dosis das ideale Körpergewicht verwendet werden.

\section{Vecuronium (z.B. Norcuron ${ }^{\circledR}$ )}

\section{Charakterisierung}

- Vecuronium ist ein nicht-depolarisierendes, mittellang wirksames Muskelrelaxans aus der Cruppe der Aminosteroide.

- Vecuronium ist zugelassen als Muskelrelaxans zur Allgemeinanästhesie bei chirurgischen Eingriffen sowie zur Erleichterung der endotrachealen Intubation.

- Die muskelrelaxierende Wirkung wird vermittelt durch kompetitive Verdrängung von Acetylcholin an den cholinergen Rezeptoren der motorischen Endplatte.

- Vecuronium ist eine polare, hydrophile Substanz.

\section{Übliche Dosierungsangaben}

- Erleichterung der endotrachealen Intubation: o, o8-o, $1 \mathrm{mg}$ i.v. pro kg KC

- Repetitionsdosis: o,o1-o,o2 mg i.v. pro kg KC

- Dosierung streng nach Wirkung!

\section{Besonderheiten bei Adipositas}

Vecuronium dringt als polare Substanz nicht ins Fettgewebe ein, sondern verteilt sich im extrazellulären Raum. Die Elimination der Substanz erfolgt vor allem hepatisch. Schwartz et al. (1991) konnten im Rahmen einer klinischen Studie keine relevanten Unterschiede bei Clearance, Verteilungsvolumen und Halbwertszeit zwischen adipösen und normalgewichtigen Patienten feststellen, wenn Vecuronium nach idealem Körpergewicht dosiert wurde. Bei Dosierung nach Gesamtkörpergewicht hingegen zeigte sich in derselben Studie eine deutliche Verlängerung der Wirkung bei adipösen $\mathrm{Pa}$ tienten.

Bei Adipositas sollte zur Berechnung der benötigten Vecuronium-Dosis das ideale Körpergewicht verwendet werden.

\section{Rocuronium (z.B. Esmeron ${ }^{\circledR}$ )}

\section{Charakterisierung}

- Rocuronium ist ein kurzwirksames, nicht-depolarisierendes Muskelrelaxans aus der Cruppe der Aminosteroide.

- Rocuronium ist zugelassen zur Erleichterung der endotrachealen Intubation sowie zur Muskelrelaxation im Rahmen der Allgemeinanästhesie bei chirurgischen Eingriffen. 
- Die muskelrelaxierende Wirkung wird vermittelt durch kompetitive Verdrängung von Acetylcholin an den cholinergen Rezeptoren der motorischen Endplatte.

- Rocuronium ist eine hydrophile quartäre Ammoniumverbindung.

\section{Übliche Dosierungsangabe}

- Erleichterung der endotrachealen Intubation: o, 6-1,o mg i.v. pro $\mathrm{kg} \mathrm{KG}$

- Repetitionsdosis: o,075-0,15 mg i.v. pro kg KC

- Dosierung streng nach Wirkung!

\section{Besonderheiten bei Adipositas}

Rocuronium liegt im Plasma als ionisierte quartäre Ammoniumverbindung vor und kann sich daher ausschließlich im Extrazellulärraum verteilen. Eine Penetration ins Fettgewebe erfolgt nicht in relevantem Ausmaß. Leykin et al. (2004) untersuchten Rocuronium an adipösen Patienten und verglichen dabei den Effekt einer Dosierung nach Gesamtkörpergewicht bzw. idealem Körpergewicht.

Dabei konnte festgestellt werden, dass bei Dosierung von Rocuronium nach Gesamtkörpergewicht bei adipösen Patienten die Wirkdauer gegenüber einer Dosierung nach idealem Körpergewicht annähernd verdoppelt war. Daher wird empfohlen, Recuronium bei Adipositas nach idealem Körpergewicht zu dosieren.

Bei Adipositas sollte zur Berechnung der benötigten Rocuronium-Dosis das ideale Körpergewicht verwendet werden.

\subsection{Antikoagulanzien}

\subsubsection{Unfraktioniertes Heparin}

\section{Unfraktioniertes Heparin (z.B. Liquemin ${ }^{\circledR}$ )}

\section{Charakterisierung}

- Unfraktioniertes Heparin ist eine Mischung heterogener, sulfatierter Glykosaminoglykane (GAG) und wird aus Schweinedarm-Mukosa isoliert.

- Unfraktioniertes Heparin inhibiert sowohl Faktor Xa als auch Thrombin im Verhältnis 1:1. Die Bindung von Heparin an Antihrombin III (AT III) steigert dessen inhibitorische Wirkung auf Serinproteasen (z.B. einige Blutgerinnungsfaktoren) um ein Vielfaches und verstärkt damit indirekt die Hemmung des Faktors Xa. Unfraktioniertes Heparin bindet aufgrund seiner Molekülgröße
(> 5,4 kDa, Kettenlänge > 17 Monosaccharide) nicht nur an AT III, sondern gleichzeitig auch an Thrombin (Faktor IIa), wodurch eine Inhibierung des Thrombins ermöglicht wird.

- Unfraktioniertes Heparin ist indiziert zur Thromboembolie-Prophylaxe, Therapie venöser und arterieller Thromboembolien und zur therapeutischen Antikoagulation bei Patienten mit extrakorporalem Kreislauf (z.B. Hämofiltration).

- Unfraktioniertes Heparin ist eine hydrophile Substanz.

\section{Übliche Dosierungsangabe}

Die Dosierung erfolgt abhängig vom Gerinnungsstatus entsprechend der gemessenen aPTT oder ACT.

Für die therapeutische Heparinisierung gilt als Faustregel:

-1 aPTT: 1,5-2,5-fach über dem Normwert

I. ACT: 400-500 Sekunden

Zur Orientierung können folgende Dosierungen eingesetzt werden:

- Initial: 1.000-2.00o IE UFH i.v., bei Lungenembolie: 5.00o IE UFH i.v. Die initiale Bolusgabe ist erforderlich, falls ein sofortiger Wirkeintritt notwendig ist (z.B. vor Hämodialyse).

- Erhaltungsdosis: 400-1.00o IE/h UFH i.v. als kontinuierliche Infusion

Für die Thromboseprophylaxe („low dose“-Heparinisierung) gilt folgende Empfehlung:

- 2-3 x 5.00o IE bis 2-3 X 7.500 IE UFH s.c.

\section{Besonderheiten bei Adipositas}

Unfraktioniertes Heparin verteilt sich als hydrophile Substanz nur sehr schlecht im adipösen Gewebe. Vielmehr hängt das Verteilungsvolumen von Heparin vom Blutvolumen ab, das bei übergewichtigen Patienten zwar insgesamt vergrößert, in adipösem Gewebe jedoch im Vergleich zum fettfreien Gewebe verringert ist. Die Gefahr von Über- bzw. Unterdosierungen ist damit gegeben, wenn bei der Überlegung der richtigen Dosierung das Cesamt- bzw. Idealgewicht berücksichtigt wird. Deshalb gibt es Empfehlungen, das Idealgewicht um 30-40\% des Übergewichts zu korrigieren (Ellison et al. 1989; Medico u. Walsh 2010; Myzienski et al. 2010; Schwiesow et al. 2005; Spruill et al. 2001; Yee et al. 1998). Folgende Berechnungsformeln für das Dosierungsgewicht ergeben sich dabei:

- Dosierungsgewicht $[\mathrm{kg}]=\mathrm{IBW}+0,3 \mathrm{x}(\mathrm{TBW}-\mathrm{IBW})$ oder

- Dosierungsgewicht $[\mathrm{kg}]=\mathrm{IBW}+0,4 \mathrm{x}(\mathrm{TBW}-\mathrm{IBW})$ 
In der Praxis sind allerdings Dosierungsangaben in IE pro kg Körpergewicht ungebräuchlich.

\section{Thromboseprophylaxe bei adipösen Patienten}

Da in der Praxis die Dosierung von unfraktioniertem Heparin zur Prophylaxe oft nicht nach dem entsprechenden Körpergewicht berechnet wird, empfehlen die Autoren für die subkutane Thromboseprophylaxe folgende Dosierung bei adipösen Patienten: $3 \mathrm{x}$ 5.000-7.50o IE s.c.

Therapeutische Antikoagulation bei adipösen Patienten Zur Behandlung thromboembolischer Erkrankungen wird beim normalgewichtigen Patienten als initiale Heparin-Gabe ein Bolus von 5.ooo IE i.v. empfohlen. Daran schließt sich eine kontinuierliche Infusion von 1.00o IE/h i.v. an. Es wird eine Erhöhung der aPTT um das 1,5- bis 2,5-fache des Normwertes angestrebt. Beim adipösen Patienten sollte bereits initial die kontinuierliche Rate höher gewählt werden. Als Orientierung dient das berücksichtigte Übergewicht. Dabei wird die kontinuierliche Rate um den Prozentsatz erhöht, der das prozentuale Verhältnis von berücksichtigtem Übergewicht zu Idealgewicht angibt.

\footnotetext{
Beispiel

Patient mit idealem Körpergewicht (IBW) von $80 \mathrm{~kg}$, tatsächliches Körpergewicht (TBW) von $180 \mathrm{~kg}$.

Berücksichtigtes Übergewicht $=0,4 \times($ TBW - IBW $)=$ $0,4 \times(180 \mathrm{~kg}-80 \mathrm{~kg})=0,4 \times 100 \mathrm{~kg}=40 \mathrm{~kg}$

Das berücksichtigte Übergewicht $(40 \mathrm{~kg})$ entspricht $50 \%$ des Idealgewichtes $(80 \mathrm{~kg})$. Daher wird die übliche kontinuierliche Laufrate des Heparins (1000 IE/h) um 50\% erhöht auf $1.500 \mathrm{IE} / \mathrm{h}$.
}

Eine Empfehlung zur prozentualen Erhöhung der initialen Bolusgabe kann nicht gegeben werden.

In jedem Fall muss eine regelmäßige Kontrolle der aPTT oder ACT erfolgen und die Dosierung entsprechend angepasst werden.

Da insbesondere bei adipösen Patienten das Risiko von VTE (venösen Thromboembolien) erhöht ist, muss auf eine ausreichende Antikoagulation geachtet werden.

Da die Wirkung des unfraktionierten Heparins an Antithrombin III (AT III) gebunden ist, sollte v.a. bei unbefriedigender antikoagulatorischer Wirkung eine Kontrolle des AT III-Spiegels erfolgen. Ein Wert der AT III-Aktivität von 60-80\% sollte angestrebt werden.

Unfraktioniertes Heparin ist aufgrund der kurzen Halbwertszeit (ca. 90-120 min) sehr gut steuerbar.
Durch Abstellen der Zufuhr unfraktionierten Heparins kann innerhalb relativ kurzer Zeit (1-2 Stunden) von einer Normalisierung der Gerinnung ausgegangen werden. Das mögliche Komplikationsrisiko infolge von Über-bzw. Unterdosierung kann deshalb relativ rasch minimiert werden. Bei Patienten mit bereits eingeschränkter Gerinnung vor Applikation von UFH oder schwerer Leber-bzw. Niereninsuffizienz muss allerdings mit einer längeren Zeitdauer bis zu einer Normalisierung der Gerinnung gerechnet werden.

Aufgrund der guten Steuerbarkeit des unfraktionierten Heparins kann eine Dosierung entsprechend der gemessenen aPTT oder ACT relativ rasch angepasst werden.

Die Dosierung erfolgt abhängig vom Gerinnungsstatus und wird entsprechend der gemessenen aPTT oder ACT angepasst. Eine regelmäßige Blutgerinnungskontrolle (mind. 1 x pro Tag) sollte insbesondere bei adipösen und/oder Patienten mit eingeschränkter Nierenfunktion erfolgen.

\subsubsection{Niedermolekulare Heparine}

\section{Enoxaparin (z.B. Clexane ${ }^{\circledR}$ )}

\section{Charakterisierung}

- Enoxaparin wird aus unfraktioniertem Heparin durch alkalische Depolymerisation des HeparinBenzylesters synthetisiert. Es besitzt ein mittleres Molekulargewicht von 4,5 kDa.

- Niedermolekulare Heparine (NMH) bestehen im Vergleich zu unfraktioniertem Heparin aus einem Anteil kürzerer Polysaccharidketten. Je kleiner das Molekulargewicht bzw. kürzer die Kettenlänge, desto mehr wird der Faktor Xa und desto geringer wird Thrombin inhibiert. Die antiFaktor Xa/anti-Thrombin-Ratio, ein analytisches Differenzierungsmerkmal innerhalb der $\mathrm{NMH}-$ Gruppe, beträgt demnach für Enoxaparin im Mittel 4,3 . Die antikoagulatorische Wirkung entsteht daher hauptsächlich in der Hemmung des Faktors Xa.

- Enoxaparin ist indiziert zur Prophylaxe tiefer Venenthrombosen, Thromboseprophylaxe bei Patienten mit extrakorporalem Kreislauf während der Hämodialyse und zur perioperativen Umstellung von Marcumar-Patienten auf Enoxaparin.

- Enoxaparin ist eine hydrophile Substanz.

\section{Übliche Dosierungsangabe}

$20 \mathrm{mg}\left(\right.$ z.B. o, $\left.2 \mathrm{ml} \mathrm{Clexane}{ }^{\circledast}\right)=2.000$ IE Anti-Faktor $\mathrm{Xa}$ 
- Thromboseprophylaxe: 1 x 20-40 mg s.c. pro Tag (beim normalgewichtigen Erwachsenen)

- Das individuelle Thromboserisiko (z.B. bei Tumorpatienten), die Art des operativen Eingriffes sowie die Dauer der Immobilisation sind bei der Wahl der Dosierung zu berücksichtigen.

- Therapeutische Antikoagulation: 2 x o, 5-1 mg/kg KG s.c. pro Tag

- Die Dosierungs-Kontrolle/Steuerung erfolgt i.d.R. abhängig vom Gerinnungsstatus entsprechend der gemessenen anti-Faktor Xa-Aktivität im Blutplasma. Die Blutentnahme erfolgt dafür 3-(4)Stunden nach s.c.-Applikation.

- Zielspiegel:

- Prophylaxe: o,2-o,4 IE anti-Faktor Xa/ml

- Therapie: o,4-o,8 IE anti-Faktor Xa/ml

\section{Besonderheiten bei Adipositas}

Die Pharmakokinetik von Enoxaparin ist bei adipösen Patienten im Vergleich zu Normalgewichtigen unterschiedlich. Es konnte gezeigt werden, dass nach einer subkutanen Enoxaparin-Gabe von 1,5 mg/ kg Gesamtkörpergewicht die AUEC (= Fläche unter der Plasma-Aktivitäts [Effektivitäts]-Zeit-Kurve) für den Faktor Xa statistisch signifikant vergrößert war. Die maximale anti-Faktor Xa-Aktivität wurde bei adipösen Patienten 1 Stunde später erreicht, was für eine langsamere Absorption von Enoxaparin spricht (Hanley et al. 2010; Sanderink et al. 2002).

Insgesamt gibt es zurzeit keinen Konsens bezüglich der optimalen Dosierungsempfehlungen von Enoxaparin bei adipösen Patienten. Bevorzugt wird nach derzeitigem Kenntnisstand die Dosierung von Enoxaparin nach Gesamtkörpergewicht bei adipösen Patienten. Die prophylaktische Gabe von Enoxaparin sollte ebenfalls Patienten-individuell und gewichtsadaptiert erfolgen, um adäquate Zielspiegel für die Thromboseprophylaxe zu erreichen (Clark et al. 2008; Hirsh et al. 2008; Medico u. Walsh 2010; Rondina et al. 2010).

Folgende Dosierungsempfehlungen können entsprechend aktuellen Publikationen gegeben werden (Bazinet et al. 2005; Hanley et al. 2010):

- Thromboseprophylaxe: 1 x o,5 mg/kg Gesamtkörpermasse s.c. pro Tag

- Therapeutische Antikoagulation: 2 x 1,o mg/kg Gesamtkörpermasse s.c. pro Tag oder 1 x 1,5 mg/kg Gesamtkörpermasse s.c. pro Tag; ab einem Gewicht von $\geq 150 \mathrm{~kg}$ kann eine Reduktion auf 2 x o,7 mg/ $\mathrm{kg}$ sinnvoll sein

Die Autoren empfehlen sowohl für die prophylaktische als auch für die therapeutische Anwendung von niedermolekularen Heparinen bei adipösen Patienten eine regelmäßige Kontrolle des Gerinnungsstatus durch Messung der anti-Faktor Xa-Aktivität, um Über- bzw. Unterdosierungen zu vermeiden.

Da insbesondere bei adipösen Patienten das Risiko von venösen Thromboembolien (VTE) erhöht ist, muss auf eine ausreichende Antikoagulation geachtet werden.

Eine regelmäßige Kontrolle des Gerinnungsstatus durch Messung der anti-Faktor Xa-Aktivität empfiehlt sich insbesondere bei adipösen Patienten. Die Blutentnahme erfolgt dafür 3 - (4) Stunden nach s.c.-Applikation. Bei Vorliegen einer Niereninsuffizienz empfiehlt sich wegen der Kumulationsgefahr eine Umstellung auf unfraktioniertes Heparin.

Zur Dosisberechnung von Enoxaparin wird bei adipösen Patienten sowohl für die Prophylaxe als auch für die Therapie laut derzeit zur Verfügung stehenden Empfehlungen das Gesamtkörpergewicht bevorzugt.

\section{Dalteparin (z.B. Fragmin ${ }^{\circledR}$ )}

\section{Charakterisierung}

- Dalteparin wird synthetisiert, indem unfraktioniertes Heparin durch Nitritspaltung depolymerisiert wird. Es besitzt ein Molekulargewicht von ca. $6 \mathrm{kDa}$.

- Im Vergleich zu unfraktioniertem Heparin bestehen niedermolekulare Heparine (NMH) aus einem Anteil kürzerer Polysaccharidketten. Je kleiner das Molekulargewicht bzw. kürzer die Kettenlänge, desto mehr wird der Faktor Xa und desto geringer wird Thrombin inhibiert. Daraus ergibt sich für Dalteparin eine anti-Faktor $\mathrm{Xa} /$ anti-Thrombin-Ratio, ein analytisches Differenzierungsmerkmal innerhalb der NMH-Cruppe, von 2,5. Die antikoagulatorische Wirkung entsteht hauptsächlich durch Hemmung des Faktors Xa.

- Dalteparin ist indiziert zur Prophylaxe tiefer Venenthrombosen, Therapie akuter tiefer Venenthrombosen und Lungenembolie.

- Dalteparin ist eine hydrophile Substanz.

\section{Übliche Dosierungsangabe}

- Thromboseprophylaxe: 1 x 2.500-5.00o IE s.c. (beim normalgewichtigen Erwachsenen)

- Das individuelle Thromboserisiko, die Art des operativen Eingriffes sowie die Dauer der Immobilisation sind bei der Wahl der Dosierung zu berücksichtigen. 
- Therapeutische Antikoagulation: 1 x 200 IE/kg KG s.c. oder 2 x $100 \mathrm{IE} / \mathrm{kg} \mathrm{KG} \mathrm{s.c.}$

- Pro Tag beträgt die maximale Gesamtdosis 18.00o IE

- Die Dosierungs-Kontrolle/Steuerung erfolgt i.d.R. abhängig vom Gerinnungsstatus entsprechend der gemessenen anti-Faktor XaAktivität im Blutplasma. Die Blutentnahme erfolgt dafür 3-(4) Stunden nach s.c.-Applikation.

- Zielspiegel:

- Prophylaxe: o,2-o,4 IE anti-Faktor Xa/ml

- Therapie: o,4-o,8 IE anti-Faktor Xa/ml

\section{Besonderheiten bei Adipositas}

Laut Fachinformation $\left(\right.$ Fragmin $^{\circledR}$ ) wird bei Patienten mit einem Körpergewicht von größer als 83 kg zur Therapie die maximale Tagesgesamtdosis von 18.0oo I.E. eingesetzt. Clark et al. (2008) verweisen allerdings auf Daten, die keine Tageshöchstdosis für die Prophylaxe bei adipösen Patienten mit hohem Risiko postulieren. Dem zugrunde liegt das erhöhte Thromboserisiko bei adipösen Patienten. Zur Berechnung der Dosierung wird entweder das Gesamtkörpergewicht (TBW) oder das Dosierungsgewicht, d.h. Idealgewicht (IBW) plus 40\% des Übergewichts, verwendet (Hanley et al. 2010; Yee JY et al. 2000). Es gilt folgende Formel:

- Dosierungsgewicht $[\mathrm{kg}]=\mathrm{IBW}+0,4 \times(\mathrm{TBW}-\mathrm{IBW})$

Die Autoren empfehlen sowohl für die prophylaktische als auch für die therapeutische Anwendung von niedermolekularen Heparinen bei adipösen Patienten eine Kontrolle des Gerinnungsstatus durch Messung der anti-Faktor Xa-Aktivität, um Über- bzw. Unterdosierungen zu vermeiden.

Da insbesondere bei adipösen Patienten das Risiko von VTE (venösen Thromboembolien) erhöht ist, muss auf eine ausreichende Antikoagulation geachtet werden.

Eine regelmäßige Kontrolle des Gerinnungsstatus durch Messung der anti-Faktor Xa-Aktivität empfiehlt sich insbesondere bei adipösen Patienten. Die Blutentnahme erfolgt dafür 3 - (4) Stunden nach S.C.Applikation. Bei Vorliegen einer Niereninsuffizienz empfiehlt sich wegen der Kumulationsgefahr eine Umstellung auf unfraktioniertes Heparin.

Zur Dosisberechnung von Dalteparin bei adipösen Patienten empfiehlt sich entweder das Gesamtkörpergewicht oder das Dosierungsgewicht, d.h. ideales Körpergewicht plus $40 \%$ des Übergewichts.

\subsubsection{Direkte Thrombininhibitoren}

Argatroban (z.B. $\operatorname{Argatra}^{\circledR}$ )

\section{Charakterisierung}

- Argatroban ist ein Arginin-Derivat, das synthetisch hergestellt wird.

- Durch direkte Hemmung von sowohl freiem als auch gebundenem Thrombin wird die Fibrinbildung inhibiert. Darüber hinaus wird die Aktivierung von Gerinnungsfaktoren (V, VIII und XIII), Protein $C$ und die Thrombozytenaggregation gehemmt. Die Wirkung von Argatroban ist unabhängig von Antithrombin III (AT III).

- Argatroban ist indiziert zurThromboseprophylaxe und -therapie bei Patienten mit Heparin-induzierter Thrombozytopenie (HIT II).

- Die Verteilung von Argatroban erfolgt zum größten Teil in die extrazelluläre Flüssigkeit.

\section{Übliche Dosierungsangabe}

Die Dosierung erfolgt abhängig vom Cerinnungsstatus entsprechend der gemessenen aPTT oder ACT.

- Initial: (o, 2-)o, $5^{-1} \mu \mathrm{g} / \mathrm{kg} \mathrm{KG} / \mathrm{min}$. i.v. (beim normalgewichtigen Erwachsenen)

- In der Praxis reichen oft Dosierungen von 1 bis $2 \mathrm{mg} / \mathrm{h}$ beim nicht-adipösen Patienten aus.

- Die Ziel-aPTT sollte in der Regel zwischen dem 1,5-3-fachen Wert des Ausgangswertes liegen, jedoch maximal $100 \mathrm{sec}$.

\section{Besonderheiten bei Adipositas}

Die Metabolisierung von Argatroban, die in der Leber stattfindet, erhöht sich mit zunehmendem Gesamtkörpergewicht (Cox et al. 2004; Medico u. Walsh 2010). Adipöse Patienten, die entsprechend ihrem tatsächlichen Gewicht die gleiche gewichtsadaptierte Dosierung wie normalgewichtige Patienten erhielten, erzielten eine vergleichbare aPTT (Rice et al. 2007; Hanley et al. 2010). Zur initialen Dosisberechnung von Argatroban empfiehlt sich deshalb das Gesamtkörpergewicht, wobei die Dosierung im weiteren Verlauf abhängig vom Gerinnungsstatus entsprechend der aPTT oder ACT angepasst werden muss.

Da insbesondere bei adipösen Patienten das Risiko von VTE (venösen Thromboembolien) erhöht ist, muss auf eine ausreichende Antikoagulation geachtet werden.

Aufgrund der kurzen Halbwertszeit (ca. 1 Stunde) ist Argatroban gut steuerbar. Das mögliche Komplikationsrisiko infolge von Über- bzw. Unterdosierung 
kann deshalb relativ rasch minimiert werden. Im Falle einer Überdosierung kann ca. 2 Stunden, bei Patienten mit eingeschränkter Leberfunktion frühestens ca. 4 Stunden, nach Abstellen der Infusion wieder eine Normalisierung der Gerinnung erreicht werden.

Die Dosierung erfolgt abhängig vom Gerinnungsstatus und wird entsprechend der gemessenen aPTT oder ACT angepasst. Eine regelmäßige Blutgerinnungskontrolle (mind. 1 x pro Tag) sollte insbesondere bei adipösen Patienten erfolgen. Bei Patienten mit mäßig eingeschränkter Leberfunktion kann eine Dosisreduktion erforderlich sein. Die Anwendung von Argatroban bei Patienten mit stark eingeschränkter Lebersyntheseleistung ist kontraindiziert. Eine Niereninsuffizienz stellt keine Kontraindikation dar.

Momentane Empfehlungen bevorzugen bei adjpösen Patienten das Gesamtkörpergewicht zur initialen Dosisberechnung von Argatroban.

\subsection{Antiinfektiva}

Unzureichend behandelte Infektionen können massive Komplikationen verursachen. Weichteilinfektionen, akutes Abdomen oder Pneumonien führen zu schweren Infektionen oder Sepsis. Voraussetzungen für den Behandlungserfolg sind operative Fokussanierung, sofern möglich, und die adäquate Antibiotikatherapie. Die üblichen Dosierungsangaben beziehen sich häufig auf das ideale Körpergewicht. Ob die applizierten Dosierungen in Anbetracht des enormen tatsächlichen Körpergewichts auch bei adipösen Patienten ausreichen, ist bisher wenig untersucht. Es gibt Empfehlungen, die zur Berechnung der Dosierung das Dosierungsgewicht bevorzugen. Dabei werden das tatsächliche Gesamtkörpergewicht (TBW), das Idealgewicht (IBW) sowie ein Antibiotikagruppenspezifischer Korrekturfaktor berücksichtigt.

- Berücksichtigtes Übergewicht $=$ Korrekturfaktor x (TBW - IBW)

- Dosierungsgewicht $=\mathrm{IBW}+$ Berücksichtigtes Übergewicht

Dabei bestimmen folgende zwei Faktoren die Festlegung des Korrekturfaktors in Abhängigkeit von den physikalisch-chemischen Eigenschaften des Antibiotikums.

- 30\% des adipösen Übergewichtes (TBW - IBW) werden von Wasser und damit von zusätzlichem Extrazellulärraum gebildet.

- Die Pharmakokinetik des Antibiotikums kann bei adipösen Patienten mit erhöhtem Fettanteil verändert sein.
Die in den folgenden Kapiteln gegebenen Empfehlungen zur Berechnung des Dosierungsgewichts gelten nach Meinung der Autoren bei Patienten mit einem BMI größer als 40.

\subsubsection{Betalaktam-Antibiotika}

Beispiele: Piperacillin/Tazobactam (z.B. Tazobac ${ }^{\circledR}$ ), Ceftazidim (z.B. Fortum ${ }^{\circledR}$ ), Meropenem (z.B. Meronem ${ }^{\circledR}$ )

\section{Charakterisierung}

- Betalaktam-Antibiotika wirken bakterizid.

- Entscheidend ist die Zeitdauer des Wirkspiegels oberhalb der minimalen Hemmkonzentration (MHK), d.h. für eine erfolgreiche Therapie ist eine Dosierung zu wählen, mit der man möglichst über einen längeren Zeitraum konstant hohe Wirkkonzentrationen erreicht. Von Vorteil ist deshalb häufig die Gabe der Gesamttagesdosis aufgeteilt in kleinere Tagesdosen.

- Betalaktam-Antibiotika besitzen eine relativ große therapeutische Breite.

- Betalaktam-Antibiotika sind hydrophile Substanzen.

\section{Besonderheiten bei Adipositas}

Betalaktam-Antibiotika, wie Penicilline, Cephalosporine und Carbapeneme, verteilen sich als hydrophile Antibiotika gut im Extrazellulärraum. Üblicherweise werden für Erwachsene die Dosierungsempfehlungen in Standarddosierungen unabhängig vom Körpergewicht angegeben. Obwohl es derzeit keinen etablierten Korrekturfaktor gibt, ist aufgrund der Verteilung im Extrazellulärraum des adipösen Gewebes jedoch empirisch ein Korrekturfaktor von o,3 naheliegend (Wurtz et al. 1997).

I. Dosierungsgewicht $=\mathrm{IBW}+0,3 \times(\mathrm{TBW}-\mathrm{IBW})$

Die Erhöhung der Tagesdosis durch Addition einer üblichen Einzeldosis erscheint in den meisten Fällen bei massiver Adipositas als der pragmatische Weg. Von Vorteil ist dabei die große therapeutische Breite der Betalaktam-Antibiotika.

Beispiel:

Patient mit tatsächlichem Körpergewicht $180 \mathrm{~kg}$ und idealem Körpergewicht $80 \mathrm{~kg}$. Standarddosierung Piperacillin: $3 \times 4 \mathrm{~g}$ i.v./Tag.

Dosierungsgewicht $=80 \mathrm{~kg}+0,3 \times(180 \mathrm{~kg}-80 \mathrm{~kg})=$ $110 \mathrm{~kg}$

Dosis: Wenn für den normalgewichtigen Erwachsenen $3 \times 4 \mathrm{~g}$ empfohlen werden, erscheint eine zusätzliche Do- 
sis für die $30 \%$ des Übergewichtes (30 kg sind ca. ein Drittel des Idealgewichtes) nicht zu hoch. Der Patient erhält somit $4 \times 4 \mathrm{~g}$ Piperacillin i.v./Tag.

Für die Berechnung der Dosierung wird empirisch ein Korrekturfaktor von 0,3 berücksichtigt. Durch Addition einer üblichen Einzeldosis wird die Tagesdosis erhöht.

\subsubsection{Chinolone (Gyrasehemmer)}

Beispiele: Ciprofloxacin (Ciprobay ${ }^{\circledR}$ ), Moxifloxacin (Avalox ${ }^{\circledR}$ )

\section{Charakterisierung}

- Chinolone besitzen eine bakterizide Wirkung.

- Die therapeutische Breite von Chinolonen ist im Vergleich zu Betalaktamen geringer. Auf Verschlechterung der Nierenfunktion und Leberwerterhöhungen insbesondere mit cholestatischer Schädigung muss geachtet werden.

\section{Besonderheiten bei Adipositas}

Chinolone verteilen sich nicht stark im Extrazellulärraum und im Vergleich zu Betalaktam-Antibiotika und Aminoglykosiden sind ihre Plasmaspiegel eher gering. Bei einem massiv adipösen Patienten ergibt sich aufgrund der guten Gewebepenetration ein größeres Verteilungsvolumen. Die Plasmaspiegel sind niedriger und fallen rascher ab als beim Normalgewichtigen, sodass von einer schnelleren Elimination ausgegangen werden muss. Darüber hinaus verteilen sich Chinolone teilweise in das Fettgewebe, weshalb ein Korrekturfaktor von o, 45 für die Berechnung des Dosierungsgewichtes empfohlen wird. (Allard et al. 1993; Erstad et al. 2004; Medico u. Walsh 2010) - Dosierungsgewicht $=\mathrm{IBW}+0,45 \times(\mathrm{TBW}-\mathrm{IBW})$

Ein pragmatischter Weg scheint in den meisten Fällen bei massiver Adipositas die Erhöhung der Tagesdosis durch Addition einer üblichen Einzeldosis zu sein.

\section{Beispiel:}

Patient mit tatsächlichem Körpergewicht $180 \mathrm{~kg}$, ideales Körpergewicht $80 \mathrm{~kg}$. Standarddosierung Ciprofloxacin: $2 \times 400 \mathrm{mg}$ i.v./Tag

$$
\text { Dosierungsgewicht }=80 \mathrm{~kg}+0,45 \times(180 \mathrm{~kg}-80 \mathrm{~kg})=
$$
$125 \mathrm{~kg}$

Dosis: Wenn für den normalgewichtigen Erwachsenen 2 x $400 \mathrm{mg}$ empfohlen werden, erscheint eine zusätzliche Dosis für die $45 \%$ des Übergewichtes ( $45 \mathrm{~kg}$ ist ca. halbes Idealgewicht) nicht zu hoch. Der Patient erhält somit $3 \mathrm{x}$ $400 \mathrm{mg}$ Ciprofloxacin i.v. pro Tag.
Die Tagesdosis sollte je nach Schweregrad der Infektion zwischen 2-3 x $400 \mathrm{mg}$ Ciprofloxacin i.v. pro Tag liegen, falls sich der Wert des berücksichtigten Übergewichts einem Viertel des Idealgewichts nähert.

Für Moxifloxacin gibt es derzeit keine dezidierten Empfehlungen.

Für die Berechnung der Dosierung wird empirisch ein Korrekturfaktor von 0,45 berücksichtigt. Durch Addition einer üblichen Einzeldosis wird die Tagesdosis erhöht.

\subsubsection{Aminoglykoside}

Beispiele: Gentamicin (z.B. Refobacin ${ }^{\circledR}$ ), Tobramycin (z.B. Gernebcin ${ }^{\circledR}$ )

\section{Charakterisierung}

- Aminoglykoside wirken abhängig von der Wirkstoffkonzentration bakterizid. Für eine erfolgreiche Therapie ist es deshalb sinnvoll, kurzfristig möglichst hohe Spitzenspiegel zu erzielen.

- Aminoglykoside besitzen einen sog. „postantibiotischen Effekt", d.h. die Vermehrung der Bakterien ist für ca. 6 bis 8 Stunden gehemmt, selbst dann, wenn keine Wirkstoffkonzentrationen nachweisbar sind. Deshalb wird eine einmalige Dosierung empfohlen. (Ausnahme: Zur Therapie der Enterokokken-induzierten Endokarditis wird die Tagesdosis in zwei bis drei Einzeldosen verabreicht.)

- Aminoglykoside sind hydrophile Substanzen.

\section{Besonderheiten bei Adipositas}

Im Allgemeinen sollte die Dosierung sowohl bei normalgewichtigen als auch bei adipösen Patienten immer entsprechend dem gemessenen Serum-Talspiegel gewählt werden (geforderter Talspiegel: < $2 \mathrm{mg} / \mathrm{l}$ ), um das potenziell oto- und nephrotoxische Risiko so gering wie möglich zu halten. Die Blutabnahme erfolgt dabei unmittelbar vor der nächsten Gabe.

Als hydrophile Substanzen verteilen sich Aminoglykoside gut im Extrazellulärraum und damit auch im adipösen Gewebe. Die Dosierungen erfolgen nach Körpergewicht, wobei für adipöse Patienten zur Berechnung das Dosierungsgewicht unter Berücksichtigung eines Korrekturfaktors von o,4 allgemein empfohlen wird, d.h. die Summe aus Idealgewicht und 40\% des Übergewichtes ergibt das Dosierungsgewicht (Wurtz et al. 1997):

- Dosierungsgewicht $=\mathrm{IBW}+0,4 \times(\mathrm{TBW}-\mathrm{IBW})$ 


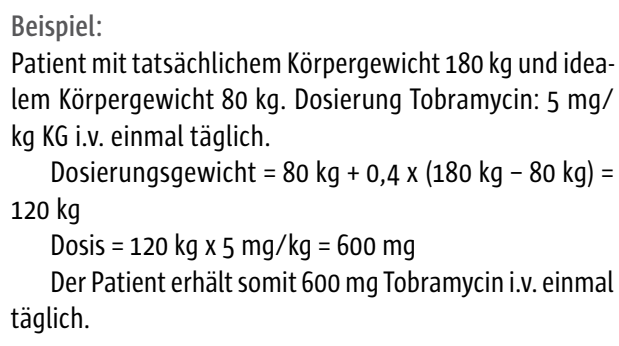

Bei bestehender Niereninsuffizienz sollte die Dosierung zur Vermeidung eines Nierenversagens zurückhaltender erfolgen. In jedem Fall sollte der Talspiegel regelmäßig kontrolliert werden.

Um das oto- und nephrotoxische Risiko zu minimieren, müssen regelmäßig die Talspiegel im Serum kontrolliert werden. Der Talspiegel wird vor der nächsten Dosis abgenommen und sollte unter $2 \mathrm{mg} / \mathrm{llie}$ gen. Ein besonders hohes Risiko besteht bei Patienten mit eingeschränkter Nierenfunktion, bei älteren Patienten, bei Dehydrierung, bei hohen Serum-Talspiegeln sowie bei einer längeren Therapiedauer (> 10 Tage).

Für die Berechnung der Dosierung wird das Dosierungsgewicht verwendet, d.h. Idealgewicht plus $40 \%$ des Übergewichts.

\subsubsection{Sonstige Antibiotika}

Für die gegen grampositive Problemkeime wirksamen Antibiotika liegen derzeit keine ausreichenden Dosierungsempfehlungen für die Anwendung bei adipösen Patienten vor, die nach Meinung der Autoren für die Praxis repräsentativ wären. Es werden deshalb im Folgenden nur kurze Hinweise bzw. Hilfestellungen gegeben.

\section{Linezolid (z.B. Zyvoxid ${ }^{\circledR}$ )}

Linezolid wirkt auf Streptokokken bakterizid, auf Staphylokokken und Enterokokken bakteriostatisch. Bei adipösen Patienten scheinen im Vergleich zum normalgewichtigen Patienten die Plasmaspiegel von Linezolid geringer zu sein. Diese können allerdings für die bakterizide Wirkung noch ausreichen (Stein et al. 2005).

\section{Vancomycin (z.B. Vancomycin HEXAL ${ }^{\circledR}$ )}

Vancomycin-Gaben sollten entsprechend der gemessenen Serum-Talspiegel erfolgen, um Überdosierungen zu vermeiden. Die Blutproben werden dafür unmittelbar vor der nächsten Applikation abgenommen.

\section{Daptomycin (z.B. Cubicin $\left.{ }^{\circledR}\right)$}

Daptomycin ist ein Lipoprotein, das nach Körpergewicht dosiert wird: 1 x 4 mg/kg KG i.v. pro Tag. Für die Berechnung der Dosierung wird das Cesamtkörpergewicht empfohlen (Dvorchik u. Damphousse 2005; Hanley et al. 2010; Medico u. Walsh 2010; Pai et al. 2007).

\subsubsection{Antimykotika}

\section{Caspofungin (z.B. Cancidas ${ }^{\circledR}$ )}

\section{Besonderheiten bei Adipositas}

Sowohl bei normalgewichtigen als auch bei adipösen Patienten beträgt die Initialdosis $70 \mathrm{mg}$ i.v. Diese Dosierung wird bei Patienten mit einem Körpergewicht größer 80 kg einmal täglich fortgeführt (1 x $70 \mathrm{mg}$ i.v. pro Tag) und nicht wie üblicherweise bei normalgewichtigen Patienten auf $50 \mathrm{mg}$ pro Tag reduziert.

\section{Fluconazol (z.B. Diflucan ${ }^{\circledR}$ )}

\section{Besonderheiten bei Adipositas}

Die übliche Standarddosierung beträgt initial $1 \mathrm{x}$ $800 \mathrm{mg}$ i.v. pro Tag, an den darauffolgenden Behandlungstagen 1 x $400 \mathrm{mg}$ pro Tag. Obwohl für adipöse Patienten derzeit keine verbindlichen Dosierungsempfehlungen vorliegen, kann folgendes Vorgehen verwendet werden:

- Patienten bis ca. $110 \mathrm{~kg} \mathrm{KG}$ (TBW):

- Initial: 1 x $800 \mathrm{mg}$ i.v. pro Tag

- Therapiefortführung: 1 x $400 \mathrm{mg}$ i.v. pro Tag

- Patienten von 110 bis $140 \mathrm{~kg} \mathrm{KG}$ (TBW):

- Initial: 1 x $800 \mathrm{mg}$ i.v. pro Tag

- Therapiefortführung: 1 x $600 \mathrm{mg}$ i.v. pro Tag

- Patienten über $140 \mathrm{~kg} \mathrm{KG}$ (TBW):

Initial: 1 x $800 \mathrm{mg}$ i.v. pro Tag

- Therapiefortführung: 1 x $800 \mathrm{mg}$ i.v. pro Tag 


\section{Literatur}

Abernethy DR, Greenblatt DJ, Divoll M, Shader RL (1983) Enhanced glucuronide conjugation of drugs in obesity: studies of lorazepam, oxazepam, and acetaminophen. I Lab Clin Med 101, 873-880

Allard S, Kinzig M, Boivin G, Sorgel F, LeBel M (1993) Intravenous ciprofloxacin disposition in obesity. Clin Pharmacol Ther 54, 368-73

Bazinet A, Almanric K, Brunet C, Turcotte I, Martineau I, Caron S, Blais N, Lalonde L (2005) Dosage of enoxaparin among obese and renal impairment patients. Thromb Res 116, 41-50

Casati A, Bignami E, Spreafico E, Mamo D (2004) Effects of obesity on wash-in and wash-out kinetics of sevoflurane. Eur | Anaesthesiol 21, 243-5

Clark NP (2008) Low-molecular-weight heparin use in the obese, elderly, and in renal insufficiency. Thromb Res 123, 58-61

Collis T, Devereux RB, Roman MJ, de Simone G, Yeh J, Howard BV, Fabsitz RR, Welty TK (2001) Relations of stroke volume and cardiac output to body composition: the strong heart study. Circulation 103, 820-5

Cox DS, Kleiman NS, Boyle DA, Aluri J, Parchman LG, Holdbrook F, Fossler M) (2004) Pharmacokinetics and pharmacodynamics of argatroban in combination with a platelet glycoprotein Ilb/IIla receptor antagonist in patients undergoing percutaneous coronary intervention. I Clin Pharmacol 44, 981-90

Dvorchik BH, Damphousse D (2005) The pharmacokinetics of daptomycin in moderately obese, morbidly obese, and matched nonobese subjects. I Clin Pharmacol 45, 48-56

Egan TD, Huizinga B, Gupta SK, Jaarsma RL, Sperry RJ, Yee JB, Muir KT (1998) Remifentanil pharmacokinetics in obese versus lean patients. Anesthesiology 89, 562-73

Ellison M), Sawyer WT, Mills TC (1989) Calculation of heparin dosage in a morbidly obese woman. Clin Pharm 8, 65-8

Erstad BL (2004) Dosing of medications in morbidly obese patients in the intensive care unit setting. Intensive Care Med 30, 18-32

Greenblatt DI, Abernethy DR, Locniskar A, Harmatz IS, Limjuco RA, Shader RI (1984) Effect of age, gender, and obesity on midazolam kinetics. Anesthesiology 61, 27-35

Hanley MJ, Darrell RA, Greenblatt D) (2010) Effect of obesity on the pharmacokinetics of drugs in humans. Clin Pharmacokinet 49, 71-87

Hirsh ], Bauer KA, Donati MB, Gould M, Samama MM, Weitz II, American College of Chest Physicians (2008) Parenteral anticoagulants: American College of Chest Physicians Evidence-Based Clinical Practice Guidelines (8th edition). Chest 133, 141S-59S

Jung D, Mayersohn M, Perrier D, Calkins I, Saunders R (1982) Thiopental disposition in lean and obese patients undergoing surgery. Anesthesiology 569, 269-74

Lemmens HI, Saidman LI, Eger El II, Laster MJ (2008) Obesity modestly affects inhaled anesthetic kinetics in humans. Anesth Analg 107, 1864-1870

Leykin Y, Pellis T Lucca M, Lomagnino G, Marzano B, Gullo A (2004) The pharmacodynamic effect of rocuronium when dosed according to real body weight ord ideal body weight in morbidly obese patients. Anesth Analg 99, 1086-9

Medico Cl, Walsh P (2010) Pharmacotherapy in the critically ill obese patient. Crit Care Clin 26, 679-688
Myzienski A, Lutz M, Smythe M (2010) Unfractionated heparin dosing for venous thromboembolism in morbidly obese patients: case report and review of the literature. Pharmacotherapy 30 105e-12e

Pai MP, Norenberg IP, Anderson T, Goade DW, Rodvold KA, Telepak RA, Mercier RC (2007) Influence of morbid obesity on the single-dose pharmcokinetics of daptomycin. Antimicrob Agents Chemother 51, 2741-7

Rice L, Hursting M), Baillie GM (2007) Argatroban anticoagulation in obese versus nonobese patients: implications for treating heparin-induced thrombocytopenia. J Clin Pharmacol 47, 1028-34

Rondina MT, Wheeler M, Rodgers GM, Draper L, Pendleton RC (2010) Weight-based dosing of enoxaparin for VTE prophylaxis in morbidly obese, medically-ill patients. Thromb Res 125, 220-3

Sanderink GJ, Liboux AL, Jariwala N, Harding N, Ozoux ML, Shukla U, Montay G, Boutouyrie B, Miro A (2002) The pharmacokinetics and pharmacodynamics of enoxaparin in obese volunteers. Clin Pharmacol Ther 72, 308-18

Schwartz AE, Matteo RS, Ornstein E, Halevy JD, Diaz I (1992) Pharmacokinetics and pharmacodynamics of vecuronium in the obese surgical patient. Anesth Analg 74, 515-8

Schwiesow S, Wessell A, Steyer T (2005) Use of a modified dosing weight for heparin therapy in a morbidly obese patient. Ann Pharmacother 39, 753-6

Servin F, Farinotti R, Haberer JP, Desmonts IM (1993) Propofol infusion for maintenance of anesthesia in morbidly obese patients receiving nitrous oxide. A clinical pharmacokinetic study. Anesthesiology 78, 657-65

Spruill WI, Wade WE, Huckaby G, Leslie RB (2001) Achievement of anticoagulation by using weight based heparin dosing protocol for obese and non-obese patients. Am J Health Sys Pharm 15, 2143-6

Stein GE, Schooley SL, Peloquin CA, Kak V, Havlichek DH, Citron DM, Tyrrell KL, Goldstein EJ (2005) Pharmacokinetics and pharmacodynamics of linezolid in obese patients with cellulitis. Ann Pharmacother 39, 427-32

Tsueda K, Warren JE, McCafferty LA, Nagle JP (1978) Pancuronium bromide requirement during anesthesia for the morbidly obese. Anesthesiology 48, 438-9

Varin F, Ducharme I, Theoret Y, Besner JG, Bevan DR, Donati F (1990) Influence of extreme obesity on the body disposition and neuromuscular blocking effect of atracurium. Clin Pharmacol Ther 48, 18-25

Wurtz R, Itokazu G, Rodvold K (1997) Antimicrobial dosing in obese patients. Clinical Infectious Diseases 25, 112-8

Yee WP, Norton LL (1998) Optimal weight base for a weight-based heparin dosing protocol. Am I Health Sys Pharm 55, 159-62

Yee JY, Duffull SB (2000) The effect of body weight on dalteparin pharmacokinetics: a preliminary study. Eur I Clin Pharmacol 56, 293-7

\section{Weiterführende Literatur}

Casati A, Putzu M (2005) Anesthesia in the obese patient: pharmacokinetic considerations. / Clin Anesth 17, 134-45

Ingrande J, Lemmens HIM (2010) Dose adjustment of anesthetics in the morbidly obese. Br | Anesth 105, i16-i23 
Schwartz AE, Matteo RS, Ornstein E, Young WL, Myers KJ (1991) Pharmakokinetics of sufentanil in obese patients. Anesth Analg 73,790-3
Darüber hinaus dienten die jeweiligen aktuellen Fachinformationen als Informationsquelle.
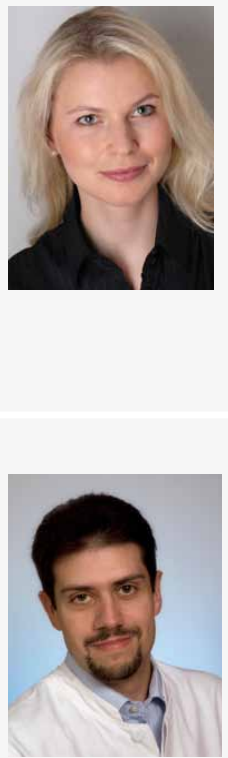

\section{Dr. rer. nat. Monika Bäumel}

Studium der Pharmazie an der Universität Regensburg und Erhalt der Approbation 2003. Im Anschluss folgte die Promotion zum Dr. rer. nat. im Fach Immunologie. Nach ihrer Tätigkeit als wissenschaftliche Mitarbeiterin am Institut für Immunologie war sie von 2007 bis 2011 als Stationsapothekerin auf den operativen und neurochirurgischen Intensivstationen des Universitätsklinikums Regensburg tätig. Ihre Schwerpunkte lagen in der Optimierung der Pharmakotherapie, Vermeidung von Interaktionen sowie Arzneimittel-induzierten Komplikationen. Seit Oktober 2011 ist sie am José-Carreras-Centrum für Somatische Zelltherapie am Universitätsklinikum Regensburg tätig.

\section{Alexander Kratzer}

Studium der Pharmazie in Regensburg und Erhalt der Approbation 2008. Anschließend Tätigkeit als Klinischer Pharmazeut in der Apotheke der Krankenhäuser des Landkreises Landshut. Seit 2010 am Universitätsklinikum Regensburg tätig als Stationsapotheker auf internistischen und operativen Intensivstationen. Daneben klinisch-pharmazeutische Forschung auf dem Gebiet des Therapeutischen Drug-Monitoring in Kooperation mit dem Institut für Pharmazie Regensburg im Rahmen eines Promotionsprojekts. 